\title{
On Wireless Sensors Communication for Overhead Transmission Line Monitoring in Power Delivery Systems
}

\author{
K.S. Hung, W.K. Lee, V.O.K. Li, K.S. Lui, P.W.T. Pong, K.K.Y. Wong, G.H. Yang, and J. Zhong \\ Department of Electrical and Electronic Engineering \\ The University of Hong Kong, Pokfulam Road, Hong Kong
}

\begin{abstract}
The transmission of energy is monitored in the smart grid through deploying sensors in all the components, including the overhead transmission lines. There are many poles/towers supporting a long overhead transmission line. Naturally, sensors are deployed on the location close to the poles/towers on each span. Due to the limited transmission range of the wireless transceiver module of a sensor, researchers generally assume that data generated by a sensor have to be delivered to the substation through a set of sensors in-between. This results in a linear network model. In this paper, we first analyze the performance of this model in handling the traffics extracted from an existing testbed. We realize that the linear network model may not be sufficient to support future smart grid applications which may have diversified requirements on data delivery. We then study a new network model in which sensor/relay nodes can also communicate with other nodes using a wide area network such as the cellular network. In this new model, the network formed can be reconfigured based on the application requirements to deliver information to the substations efficiently and effectively.
\end{abstract}

\section{INTRODUCTION}

Generally speaking, the power grid can be divided into the transmission grid and the distribution grid. The transmission grid involves the stepping up of the voltage of the power generated from the power plant and the transmission of the power to the substations through some high voltage overhead transmission lines. On the other hand, the distribution grid involves the step down of the voltage of the power in the substations and the distribution of power to different customers through low voltage power lines. Currently, many sensors have been deployed in various parts of the grid for the purpose of Supervisory Control And Data Acquisitions (SCADA) or meter readings. However, as the energy generation in a future smart grid is more dynamic and distributed, energy transmission monitoring has to be automatic with a fast response time. It is expected that different kinds of sensors will be placed everywhere in the whole grid to collect information. Some information may carry urgent messages that must be delivered to the control center immediately, while some information is less important. Different kinds of information require different ways to be delivered for efficient monitoring and control. In this paper, we are specifically interested in how the data obtained can be transmitted to appropriate places promptly in the transmission grid for future smart grid applications.

There are several major components in the transmission grid: control center, substations, transmission lines and cable poles/towers. A transmission line goes between two substations. However, due to the long distance, there are cable poles/towers sitting between the two substations to support the cable as shown in Figure 1. Traditionally, the communications among various components in the transmission grid are very limited. The control center collects information from the substations every few seconds, usually through some slow private lines, such as, dialup modem link. Due to the improvement in technology, it is expected that more and more power companies will use much higher bandwidth, lower latency communication lines, e.g., optical fiber, to form their backbone networks [1], [2].

However, many places in the transmission grid are impossible or too costly to deploy optical fibers. A wireless solution is thus sought [3]. The concept of using wireless sensors to support substation automation has been proposed in [4]. The authors assume sensors are deployed near substations only, and different sensors do not communicate with each other. Yang et al. [5], [6] are known to be the first to propose the use of sensor networks to monitor overhead transmission lines. Sensors are put on different positions on a transmission line. Some sensors are closer to the substations while some are farther away. Sensors that are not directly connected to a substation send their data to a nearby sensor which is closer to the substation. Information can then be delivered in a hop-by-hop manner to the substations. In this case, the conditions of the portion of the transmission line located far away from the substations can also be observed. Yang et al. further implement a prototype of the power line sensor to demonstrate its feasibility in [7], and predict the real-time overload capacity of the line locally in [8], [9].

Nevertheless, they did not study how the sensors are connected to each other but assume that an underlying network is present and is formed automatically for data forwarding. To the best of our knowledge, Chen et al. [10] and Leon et al. [11] are the first to propose a network model tailor-made for supporting the overhead transmission lines monitoring applications. They suggest that each pole is equppied with a relay node, which has both short-range and long-range communication modules. Sensors are deployed near the pole and possess short-range communication ability to send their data to the relay node on the pole. The long-range communication in a relay node allows it to send the collected information to the relay on a nearly pole that is closer to the substations. In this paper, we refer this network model as linear network model. Since this model is practical, it is widely adopted in the literatures, such as, supporting the "sag" level measurement in [12] and 
supporting the adaptive de-icing scheme in [13].

On the other hand, there are several studies on the performance issue of using wireless technology under various electric power grid environment. In [7], Yang et al. experimentally test the wireless transmission quality over a high voltage transmission cable, while in [14], Gungor et al. study the wireless transmission quality experimentally in various locations of electric distribution facilities. Instead of considering the performance issue over a single link, Gumbo and Muyingi [15] investigate the performance issue of the linear network model. They find that the successful delivery ratio of the packets from those nodes far away from the substation is much less than those nodes near the substation because packets from a farther node have to travel a longer distance and the rate of collision is higher.

In this paper, we collect some realistic traffic information provided by the power grid testbed in [16]. Then, we analyze the performance of the linear network model in supporting these traffics. From the analysis, we realize that the linear network model is insufficient in supporting the needs of some possible future smart grid applications. This motivates us to develop another network model to provide a certain level of quality of services, such as, energy efficiency, latency constraints, reliability, etc. Note that these quality of service requirements are essential in supporting the future smart grid applications as different kinds of sensors and applications with different quality of service requirements are expected to coexist in the same network in the future.

This paper is organized as follows. Section II revisits the linear network model. Section III discusses some realistic traffics extracted from an existing power transmission line monitoring application testbed [16]. Then, we elaborate the problem of supporting these traffics in the linear network model. Afterwards, a new network model is proposed so as to provide a certain level of quality of services in Section IV. Finally, we will conclude our work with some future directions in Section V.

\section{LINEAR NETWORK MODEL}

In this section, we will discuss the linear network model proposed in [11]. Figure 1 shows an example of a long overhead power transmission line where there are a number of supports (poles/towers) in-between. The distance between two substations can be as far as 50 kilometers. On the other hand, the distances between two poles/towers can be $0.5-1$ kilometer depending on geographical constraints and actual needs. In other words, there can be $50-100$ poles/towers between two substations.

To transmit the sensing data back to the substation, wireless communication is likely to be the most cost-effective way in terms of the equipment installation cost and the initial installation time [3]. There are many wireless communication standards [17], which can be categorized based on their theoretical communication range, maximum throughput, power consumption, wireless channel contention requirement, etc. Typically, the longer the communication range, the lower the

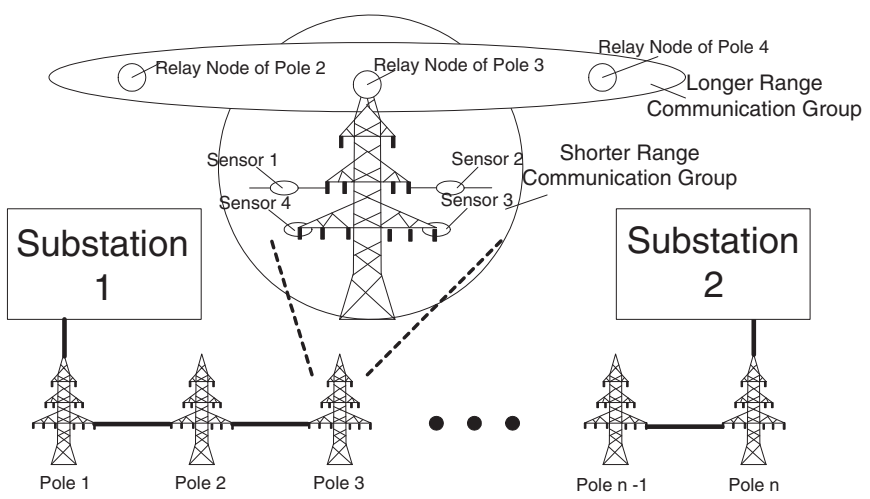

Fig. 1. An overhead transmission line example

maximum throughput and the larger the power consumption are. At the same time, to achieve the long distance transmission, a dedicated channel is generally necessary due to the possibly high interferences caused by the high transmission power. However, an exclusive use on the channel typically incurs charges in terms of the subscription fee or the license fee. Table I summarizes the characteristics of some commercially available wireless technologies [18][19][20].

In transmission line monitoring applications, sensors are usually deployed close to the poles/towers on each span [11]. A relay node is deployed on the pole/tower to collect the information from the sensors nearby and transmit the data back on behalf of these sensors. For the short range communication between sensor nodes and the relay node, which is typically less than 100 meters, Bluetooth, ZigBee, or optical fiber can be used cost-effectively. However, for the long range communications between relay nodes, which is typically longer than 400 meters, a longer range wireless communication technology has to be adopted. The work in [11] does not suggest any wireless communication suitable for this range. Yang et al. suggest the use of ZigBee Pro due to its low power consumption, acceptable throughput rate, and its ability to transmit up to $1.5 \mathrm{~km}$ in an open area [7]. However, this transmission range can probably allow a relay node to communicate with its two nearby relay nodes only. Hence, data has to be sent in a hop-by-hop manner to the substation.

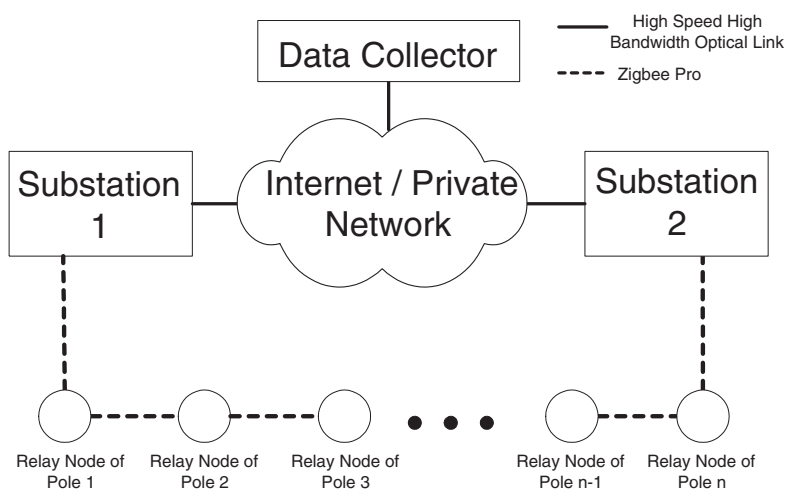

Fig. 2. High Level Abstraction of the Linear Network Model 


\begin{tabular}{|l|l|l|l|l|}
\hline Properties & Cellular Network & ZigBee & WLAN & Bluetooth \\
\hline Transmission Range & $100 \mathrm{~m}-10 \mathrm{~km}+$ & $10 \mathrm{~m}-1.5 \mathrm{~km}$ & $100 \mathrm{~m}-250 \mathrm{~m}$ & $10 \mathrm{~m}-100 \mathrm{~m}$ \\
\hline Throughput & $64 \mathrm{kpbs}(2 \mathrm{G})-384 \mathrm{kpbs}(3 \mathrm{G})$ & $20 \mathrm{kbps}-250 \mathrm{kbps}$ & $11 \mathrm{Mbps}(802.11 \mathrm{~b})-54 \mathrm{Mbps}(802.11 \mathrm{a} / \mathrm{g})$ & $3 \mathrm{Mbps}$ \\
\hline Power Consumption & High & $\begin{array}{l}1 \text { (standard range) } \\
\sim 4-5 \text { (extended range) }\end{array}$ & $\sim 50$ & $\sim 10$ \\
\hline Wireless Channel Contention & No & Yes & Yes & Nes \\
\hline Subscription Fee & Yes & No & No & No \\
\hline
\end{tabular}

${ }^{1}$ The numerical value presented in this row is relative to that of ZigBee.

TABLE I

SUMMARY OF SOME EXISTING COMMERCIAL WIRELESS COMMUNICATION TECHNOLOGIES.

Figure 2 shows a high level abstraction of the linear network model based on the following assumptions.

1) The substations are connected to the data collector server through a high speed low latency network. A large number of data can be transmitted to the data collector within a negligible amount of time.

2) A relay node is relatively more powerful than a normal sensor node in terms of energy, computational power, transmission capability, etc. However, since it is responsible for communicating with large number of nodes most of the times, it is the bottleneck in terms of network lifetime or latency.

The substations can be further modeled as a single sink node, a node that is the destination of all the data, in the network as shown in Figure 3. We further assume that there are $n$ relay nodes between the two substations. For the ease of discussion, we label these nodes as Node 1, Node 2, ... Node $n$, where Node 1 and Node $n$ are directly connected to a substation.

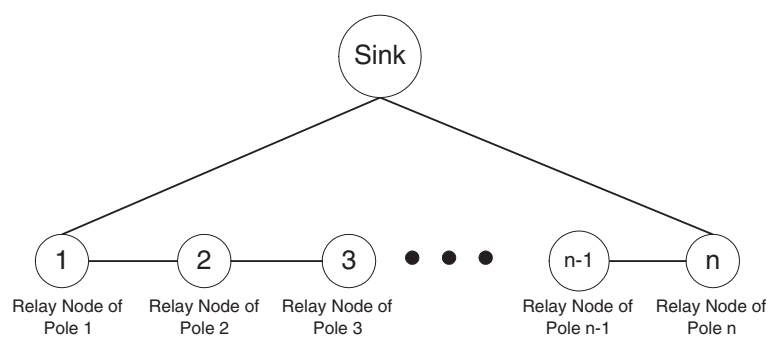

Fig. 3. Linear Network Model

\section{Performance of the Linear Network Model}

In this section, we will study the performance of the linear network model in delivering the data generated by the power grid testbed in [16]. To monitor a span of the power transmission line, several sensors have been installed, such as, accelerometers, magnetic field sensors, strain sensors, temperature sensors, etc. Note that our model works with other types of sensors, including video cameras.

Table II summarizes the types of sensors and the associated traffics in monitoring one span of the transmission line. All the sensors, except the temperature sensor, require two channels to collect data in the $x$ and $y$ dimensions. The data sampling frequency is $500 \mathrm{~Hz}$ and the data collection frequency of $2 \mathrm{~Hz}$.
In other words, the sensors will store 250 data points, and each sends out data to the relay in every $0.5 \mathrm{~s}$.

\begin{tabular}{|c|c|c|c|c|}
\hline $\begin{array}{l}\text { Type of Sen- } \\
\text { sor }\end{array}$ & $\begin{array}{l}\text { Number } \\
\text { of } \\
\text { Sensors }\end{array}$ & $\begin{array}{l}\text { Number } \\
\text { of Chan- } \\
\text { nels per } \\
\text { Sensor }\end{array}$ & $\begin{array}{l}\text { Size of each } \\
\text { sample per } \\
\text { Channel }\end{array}$ & $\begin{array}{l}\text { Total Size } \\
\text { in data } \\
\text { collection per } \\
\text { monitoring } \\
\text { cycle }\end{array}$ \\
\hline Accelerometer & 4 & 2 & $\begin{array}{l}4 \text { Bytes }(1 \\
\text { Float) }\end{array}$ & $8 \mathrm{kBytes}$ \\
\hline $\begin{array}{l}\text { Magnetic } \\
\text { Field Sensor }\end{array}$ & 2 & 2 & $\begin{array}{l}4 \text { Bytes (1 } \\
\text { Float) }\end{array}$ & 4kBytes \\
\hline $\begin{array}{l}\text { Strain } \\
\text { Sensor }\end{array}$ & 1 & 2 & $\begin{array}{l}4 \text { Bytes }(1 \\
\text { Float) }\end{array}$ & 2kBytes \\
\hline $\begin{array}{l}\text { Temperature } \\
\text { Sensor }\end{array}$ & 1 & 1 & $\begin{array}{l}4 \text { Bytes (1 } \\
\text { Float) }\end{array}$ & $1 \mathrm{kBytes}$ \\
\hline
\end{tabular}

TABLE II

SUMMARY OF THE SENSORS INFORMATION IN THE POWER GRID TESTBED IN [16] WITH THE DATA SAMPLING FREQUENCY OF 500HZ AND DATA COLLECTION FREQUENCY OF $2 \mathrm{~Hz}$.

The data sampled by each sensor suggested in Table II is unprocessed. Due to the recent advancement of the computational processing power of the sensors, it is expected that the sensors/relay node can preprocess the raw data so as to extract some important information that we are interested. In this work, we assume that even the sensors do not process the raw data, when the relay node receives them, it can extract some important attribute information inside and send it back to the substations. Table III illustrates the list of the attributes that are important to the monitoring application. The size of the processed data (i.e., $4 \mathrm{kBytes}$ of data), which is sent out by each relay node during each monitoring cycle, is approximately a quarter of that of the unprocessed data (i.e., $16 \mathrm{kBytes}$ of data).

The ability to process the data allows the power grid communication system to be intelligent enough to generate different kinds of traffics during different situations. Basically, the following scenarios are expected to occur in future smart grid applications:

1) Normal: the control center may convey the status of the transmission line in a period of $10-15$ minutes. This traffic can enjoy a loose delay tolerance of 30 s or more.

2) User-Specified: the control center may be interested in the status information of a specific span in the transmission line. The data collection rate is user-specified, and can be as fast as the data collection frequency in the relay node 


\begin{tabular}{|l|l|l|}
\hline $\begin{array}{l}\text { Monitoring } \\
\text { Parameters }\end{array}$ & Type of Sensor & Total Size \\
\hline Inclination & Accelerometers & 16 Bytes (4 Floats) \\
\hline Cable Tilt & Accelerometers & 8 Bytes (2 Floats) \\
\hline Magnetic Field & $\begin{array}{l}\text { Magnetic Field Sen- } \\
\text { sors }\end{array}$ & 16 Bytes (4 Floats) \\
\hline Cable Position & Accelerometers & 8 Bytes (2 Floats) \\
\hline Extension \& Strain & Strain Sensor & 8 Bytes (2 Floats) \\
\hline Temperature & Temperature Sensor & 4 Bytes (1 Float) \\
\hline Current & $\begin{array}{l}\text { Magnetic Field Sen- } \\
\text { sors }\end{array}$ & 4 Bytes (1 Float) \\
\hline Power Quality Graph & $\begin{array}{l}\text { Magnetic Field Sen- } \\
\text { sors }\end{array}$ & $\begin{array}{l}\text { 4kBytes (4 Floats } \times \\
\text { 250 Data Points) }\end{array}$ \\
\hline
\end{tabular}

TABLE III

SUMMARY OF THE PROCESSED INFORMATION IMPORTANT TO THE MONITORING APPLICATION

(i.e., $0.5 \mathrm{~s}$ or more in this testbed). The delay tolerance is also user-specified. (i.e., limited by the fastest possible way to deliver the traffic to the substation.)

3) Urgent: the relay node may realize that some special events are happening based on the sensor data. A warning message should be delivered together with the processed data of this span to the control center with a rate of seconds, and as soon as possible.

4) Trace-back: when the control center receives a warning message initiated by a relay node, it maybe interested in the unprocessed data collected before and after the special event happened for further analysis. This may involve the delivery of 30s or more unprocessed data to the control center as soon as possible.

5) Backup: the control center may be interested in all the unprocessed data of any span of the transmission line for future analysis purpose. This may involve huge volume of data with very loose delay tolerance.

Suppose the linear network model discussed in Section II is used. There are 100 relay nodes in-between two substations. In other words, $n=100$. Each relay node is equipped with the ZigBee Pro wireless module. (i.e., data rate of $250 \mathrm{kbps}$ $=31.25 \mathrm{kBytes}$ per second). In the subsequent description, we use data_rate to denote the data rate of the wireless module. Since the wireless channel is shared, it requires a technique for collision avoidance. The most common one is CSMA/CA. According to [21][22], the average CSMA/CA time is around $41 \mathrm{~ms}$. Therefore, the delay in transmission can be estimated by $T_{d}+T_{t}$, where $T_{d}$ represents the total time spent in CSMA/CA, and $T_{t}$ represents the total time spent in transmitting the data. $T_{d}$ can be further estimated by $n_{p} \times 41 \mathrm{~ms}$, where $n_{p}$ denotes the number of poles/towers from the source of data to the substation. Suppose $S_{d}$ denotes the size of data per towers/poles, $T_{t}$ of the data that needs to go through $n_{p}$ hops can be estimated by $\frac{S_{d} \times n_{p}}{\text { data } r a t e}$. The relay nodes should send their data to either one of the substations. Assuming each substation handles half of the relay nodes, then $n_{p}=100 / 2=50$. We further assume that to reduce messages, a node will send its data together with the data it received from its neighbor in a single message instead of multiple ones. Then, the delay of the data tranmission can be approximated by $\frac{41}{1000} \times 50+\frac{4 \times(1+\ldots+50)}{31.25}=165.25 \mathrm{~s} \sim$ 3 minutes. The information may not be up-to-date when it reaches the substations.

Under the same settings, we now analyze how much time it takes for Node 50 to send an urgent message of size $4 \mathrm{kBytes}$ to the substation. Assuming the data is sent back to the substation alone, the delay can be approximated by $\frac{41}{1000} \times 50+\frac{4 \times 50}{31.25}$ $=8.45 \mathrm{~s}$. Note that the SCADA cycle is just around $4-8 \mathrm{~s}$ [11]. It means that the delay of the urgent message can be 2 times larger than that of the SCADA cycle period. The delay approximated here can also serve as an estimate of the fastest possible user-specified delay setting on monitoring a particular span of the transmission line.

Let us move forward to consider the performance of the linear network model in handling the traceback traffic. Again, we assume Node 50 would like to transmit 30 s of unprocessed data to the substation. The volume of the traffic is $16 \times 30 \times 2=$ $960 \mathrm{KBytes}$, and the delay can then be estimated by $\frac{41}{1000} \times 50+$ $\frac{960 \times 50}{31.25}=1538.05 \mathrm{~s} \sim$ half an hour. The delay may affect the reaction time to urgent incidents.

We now consider the case of gathering data from all of the relay nodes again. All the data sensed by Node 2 to Node 50 have to go through Node 1 before reaching the substation. It is not difficult to find that Node 1 is handling $4 \times 50=200$ kBytes of data, while Node 50 is just handling 4 kBytes of data. In other words, Node 1 is handling a traffic volume 50 times that of Node 50. This uneven loading issue becomes particularly serious when the network formed is expected to work over months or even years.

Finally, we would like to consider the node failure scenario. Suppose Node 25 and Node 75 are out of services. In this case, we have no way to figure out the situations of the spans located in-between these two relay nodes even though they may work well. Also, we may not be able to figure out the exact location of failures.

\section{Proposed Network Model - Reconfigurable NeTwORK MODEL}

Although our examples in Section III may overestimate or underestimate the performances of the linear network model in the actual situations which may, in fact, depend on many other factors, these examples do give us insight on several issues that are important in designing the network model to support transmission line monitoring applications. They are delay tolerance, energy efficiency, and reliability. Since the message generated in the middle of the network has to go through a long chain of relay nodes, the delay is expected to be large. At the same time, if all the relay nodes are expected to generate a certain number of messages, the relay nodes near the substations are expected to use up a lot more energy than those in the middle of the network in the long run. Also, relay nodes failure in the network may cause an unexpected large area loss of status information.

All the issues aforementioned can be remedied if we can select an appropriate way to deliver information based on the 
traffic requirements and resource constraints. To offer a relay an alternative way to deliver its data, a relay node is equipped with several kinds of communication devices for different ranges of communication. For instance, Bluetooth, ZigBee Pro, and GSM/GPRS/UMTS ${ }^{1}$. The GSM/GPRS/UMTS device is turned on only when necessary. Therefore, unlike that of [10], [11], [15] in which each relay node can only communicate with its nearby neighbors, in our model, a relay node can turn on the GSM/GPRS/UMTS device so that it can transmit the data directly to the data collector through the cellular network. Some commercial sensors have already equipped with similar devices, such as, Power Line Sensor [24] for the temperature measurement and Sagnometer [25] for the sag level measurement of the line.

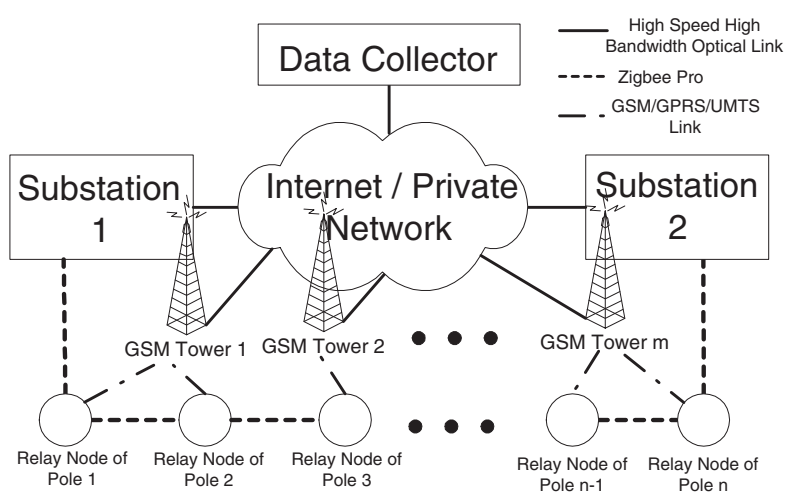

Fig. 4. High level abstraction of the Reconfigurable Network Model

Although a relay node can send out its data through different ways, there is no study on how to select among these communication options. We are the first to study a reconfigurable network model on the overhead transmission line based on traffic characteristics and resource constraints. Our model does not assume a particular long-range wireless communication technology to be used. However, for the ease of discussion, we use cellular network to refer to a network that allows a longer distance of transmission. Figure 4 shows a high level abstraction of the reconfigurable network model proposed. Each relay node can send its data to the GSM tower directly, and the GSM towers are connected to the Data Collector server with low latency, high bandwidth, low cost links. Hence, substations and GSM towers can be modeled as a single sink node in the network where the sensed data is destined for as shown in Figure 5. In the figure, relay nodes are also labeled in ascending order of their hop count to substation 1 as $1, \ldots, n$. To model the new communication path, there is a link between the relay node and the sink. However, the communicating cost and energy cost of this link

${ }^{1}$ IEEE 802.11y [23] is the newly approved standard in September 2008 adapted from IEEE $802.11 \mathrm{a}$ with the ability to transmit the signal to $5 \mathrm{~km}$ with $54 \mathrm{Mbps}$ in the licensed frequency band $3.7 \mathrm{GHz}$. However, it requires very high transmit power, specially designed antenna, and a license from the FCC (Currently not supported in countries other than US [23]). Therefore, we do not consider this technology in this work. Nevertheless, it maybe a promising wireless technology in integrating into the reconfigurable network model that we proposed here. are very high when compared to the link between the relay nodes. On the other hand, transmitting data to the sink node through a set of nodes in-between requires less cost, but this approach generally incurs higher delay and causes unbalance energy depletion in the network. As a result, a certain tradeoff exists.

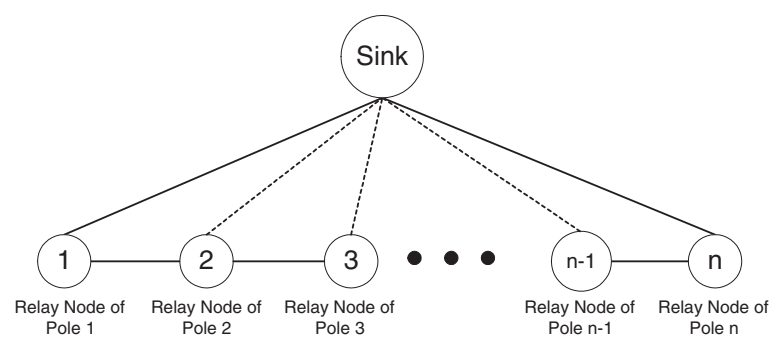

Fig. 5. Proposed Reconfigurable Network Model

We need a mechanism to send traffic to the data center that strikes a balance between delay and cost. For example, instead of turning on all GSM devices of all relays, we turn on two of them. Specifically, the delay is expected to be reduced if we turn on the devices in Nodes 33 and 66, and the traffics of the nearby relay nodes flow to these nodes instead of going through a long way to the substations. To further illustrate this issue, let us assume that Nodes $33, \ldots, 49$ will use Node 33 for message relaying, while Nodes $50, \ldots, 66$ will use Node 66 for message relaying. On the other hand, Nodes $1, \ldots, 32$ will send messages to the substation through Node 1 , while Nodes $67, \ldots, 100$ will send messages to the substation through Node 100. We further assume that GPRS is used in Nodes 33 and 66 , and the data rate is $64 \mathrm{kbps}$ ( $8 \mathrm{kBytes}$ per second). In this case, the time it takes for the data of nodes 33 to 49 to reach the data collector would be the sum of the time in transmitting the data to Node 33 and the time in transmitting the data back to the data collector by Node 33. For the first part, we can still use the formula discussed in the previous section for estimation, i.e., $\frac{41}{1000} \times 16+\frac{4 \times(1+\ldots+16)}{31.25}=18.064 \mathrm{~s}$. For the second part, note that Node 33 will also put in its own data. Therefore, the amount of information to be sent is $(16+1=17) \times 4=68 \mathrm{kBytes}$. As the rate of transmission is $8 \mathrm{kBytes}$, the time it takes is $\frac{68}{8}=8.5 \mathrm{~s}$. By summing up these two values, the estimated delay for using Node 33 (also the same for Node 66 ) will become 26.564s. Note that Nodes 67 to 100 and Nodes 1 to 32 use Node 100 and Node 1 to send data back to the substation, respectively. We also need to consider their delay in this model. The delay can be estimated by $\frac{41}{1000} \times 33+\frac{4 \times(1+\ldots+33)}{31.25}=73.161 \mathrm{~s}$. Now, we compare the maximum delay of the two models. In our model, the delay of data initiated from Node 67 is the highest. The data has to travel in a hop-by-hop manner to the substation from Node 67 through Node 100. The time it takes is 73.161s. Compared to the time it needs in the linear network model, which is $165.25 \mathrm{~s}$, our model reduces the delay by more than half.

In our model, an urgent message from a relay node can be transmitted back to the substation almost instantly by 
turning on its GPRS interface. More specifically, it requires approximately $\left(\frac{4}{8}=0.5 s\right)+$ initialization delay in transmitting the data back to the substation during urgent scenario. Since it is assumed that the initialization delay is less than a few seconds, the maximum delay in the urgent event is expected to be less than that of the linear network model. Besides, it is expected that several nearby spans should experience similar event. Therefore, these relay nodes can collaborate to turn on one GSM/GPRS/UMTS device in-between so as to balance the delay and cost issue.

On the other hand, if we turn on Node 50 or Node 51 occasionally for backing up the information in the whole network, some of the information can flow to the substations through this node instead of those near the substations. As a result, the energy depletion in different relay nodes can be much more balanced. Finally, the network can still provide information on most spans even two or more relay nodes fail due to hardware problem or multiple link failures due to transient ambient disturbances.

We mentioned earlier that the cellular link requires more energy and a higher communication cost. Currently, sensors can acquire energy through solar panels or the magnetic field around the transmission line [26]. According to the current pricing model of cellular networks, it is expected that the monetary cost of the cellular link would be related to the amount of information going through this link. We believe both energy and communication cost should be factors in determining which cellular links to be turned on.

\section{CONCLUSion AND Future WORK}

In this paper, we analyze the performance of the existing linear network model and identify its insufficiencies in supporting future smart grid applications. To deal with this issue, we propose the extension of the wireless communication capability of the relay nodes/sensors so that they can communicate with another relay node/sensor located far away. In the future, we plan to study the performance issue of this newly proposed network model against some realistic traffics. We also want to develop intelligent mechanisms to configure the network based on traffic demand, energy and communication cost.

\section{REFERENCES}

[1] G. N. Ericsson, "Classification of power systems communications needs and requirements: Experience from case studies at swedish national grids," Transaction on Power Delivery, vol. 17, no. 2, April 2002.

[2] — , "Communication requirements - basis for investment in a utility wide-area network," Transaction on Power Delivery, vol. 19, no. 1, January 2004.

[3] V. C. Gungor and F. C. Lambert, "A survey on communication networks for electric system automation," Computer Networks, vol. 50, pp. 877897, 2006.

[4] M. Nordman and M. Lehtonen, "A wireless sensor concept for managing electrical distribution networks," in IEEE Power Systems Conferenc, October 2004, pp. 1198-1206.

[5] Y. Yang, D. Divan, R. G. Harley, and T. G. Habetler, "Power line sensornet - a new concept for power grid monitoring," in IEEE Power Engineering Society General Meeting, 2006.

[6] Y. Yang, F. Lambert, and D. Divan, "A survey on technologies for implementing sensor networks for power delivery systems," in IEEE Power Engineering Society General Meeting, 2007.
[7] Y. Yang, D. Divan, R. G. Harley, and T. G. Habetler, "Design and implementation of power line sensornet for overhead transmission lines," in IEEE Power Engineering Society General Meeting, September 2009.

[8] Y. Yang, R. G. Harley, D. Divan, and T. G. Habetler, "Thermal modeling and real time overload capacity prediction of overhead power lines," in IEEE SDEMPED, August-September 2009.

[9] — , "Overhead conductor thermal dynamics identification by using echo state networks," in Proceedings of International Joint Conference on Neural Networks, June 2009.

[10] J. Chen, S. Kher, and A. K. Somani, "Energy efficient model for data gathering in structured multiclustered wireless sensor networks," in IEEE International Performace, Computing, and Communications Conference (IPCCC), April 2006.

[11] R. A. Leon, V. Vittal, and G. Manimaran, "Application of sensor network of secure electric energy infrastructure," IEEE Transactions on Power Delivery, vol. 22, no. 2, April 2007.

[12] P. Ramachandran, V. Vittal, and G. T. Heydt, "Mechanical state estimation for overhead transmission lines with level spans," IEEE Transactions on Power Delivery, vol. 23, no. 3, August 2008.

[13] Q. Huang, Y. Chen, and C. Zhang, "Design of an adaptive on-load deicing scheme for overhead power transmission line," in IEEE Power Engineering Society General Meeting, September 2009.

[14] V. C. Gungor, B. Lu, and G. P. Hancke, "Opportunities and challenges of wireless sensor networks in smart grid - a case study of link quality assessments in power distribution systems," Accepted to IEEE Transactions on Industrial Electronics.

[15] S. Gumbo and H. N. Muyingi, "Performance investigation of wireless sensor network for long distance overhead power lines; mica2 motes, a case study," in Third International Conference on Broadband Communications, Information Technology \& Biomedical Applications (Broadcom), 2008.

[16] P. W. T. Pong, K. S. Hung, R. C. L. Li, S. C. F. Tam, W. K. Lee, K. K. Y. Wong, and K. S. Lui, "A laboratory setup of a power transmission line system scaled model for testing and validation of sensor network applications," Technical Report TR-2010-002, Department of Electrical and Electronic Engineering, The University of Hong Kong, April 2010. [Online]. Available: http://www.eee.hku.hk/research/technical_reports.html

[17] Y.-K. Kwok and V. K. N. Lau, Wireless Internet and Mobile Computing: Interoperability and Performance. John Wiley \& Sons, September 2007.

[18] J. A. Gutierrez, D. B. Durocher, B. Lu, R. G. Harley, and T. G. Habetler, "Energy evaluation goes wireless: Applying wireless sensor network in industrial plant energy evaluation and planning systems," Industry Application Magazine, vol. 13, no. 2, pp. 17-23, Feburary 2007.

[19] IEEE 802.15.4-2006, "IEEE Standard for information technology-Part 15.4: Wireless medium access control (MAC) and physical layer (PHY) specifications for low rate wireless personal area networks (LRWPANS)," 2006.

[20] IEEE 802.11-2005, "IEEE Standard for information technology LAN/MAN - Specific requirements - Part 11: Wireless LAN medium access control (MAC) and physical layer (PHY) specifications," 2005.

[21] V. Shnayder, M. Hempstead, B. Chen, G. W. Allen, and M. Welsh, "Simulating the Power Consumption of Large-Scale Sensor Network Applications." in Proceedings of the 2nd international conference on Embedded networked sensor systems (ACM Sensys)., November 2004, pp. 188-200.

[22] — "PowerTOSSIM: Efficient Power Simulation for TinyOS Applications," in http://www.eecs.harvard.edu/ shnayder/ptossim, 2008.

[23] IEEE 802.11y-2008, "IEEE Standard for information technology LAN/MAN - specific requirements - Part 11: Wireless LAN medium access control (MAC) and physical layer (PHY) specifications (amendment 3," 2008.

[24] Protura, "Power line sensor," 2009. [Online]. Available: http://www.protura.no

[25] I. EDM International, "Sagnometer," 2009. [Online]. Available: http://www.edmlink.com/

[26] R. Moghe, Y. Yang, F. Lambert, and D. Divan, "A scoping study of electric and magnetic field energy harvesting for wireless sensor networks in power system applications," in IEEE Energy Conversion Congresss \& Expo, September 2009. 